\title{
PROOF OF THE INDEX CONJECTURE IN HOFER GEOMETRY
}

\author{
YASHA SAVELYEV
}

\begin{abstract}
Let $\gamma$ be an Ustilovsky geodesic and $H$ its generating function. We give a simple proof of a generalization of the conjecture stated in [7], relating the Morse index of $\gamma$, as a critical point of the Hofer length functional, with the Conley Zehnder index of the extremizers of $H$, considered as periodic orbits.
\end{abstract}

\section{Introduction}

There has not been much study of the Morse index of geodesics for the Hofer length functional on path spaces of the group of Hamiltonian diffeomorphisms $\operatorname{Ham}(M, \omega)$. Maybe this is because the problem of Morse theory for the Hofer length functional seems completely hopeless. This is possibly true to a large extent, however in [7] we showed that doing Morse theory for the Hofer length functional "virtually" can give some interesting results in symplectic topology.

In the special case where $\gamma$ is an $S^{1}$-subgroup in $\operatorname{Ham}(M, \omega)$ generated by a Morse Hamiltonian $H$, a key point in [7] was using a relationship of the Morse index of $\gamma$ with the Conley-Zehnder index of the linearized flow at the extremizers of $H$, in some special cases.

Remark 1.1. We did not use the words Conley-Zehnder index in [7], but rather the index of a certain Cauchy-Riemann operator, but this could be directly related to the above $\mathrm{CZ}$ index.

Indeed as a byproduct we arrived at the conjecture that the two indexes must coincide. A lower bound for the Morse index in terms of the Conley-Zehnder index was proved by Karshon-Slimowitz in [2] by constructing a beautiful explicit local family of shortenings of $\gamma$. Here we give a simple proof of the conjecture for more general Ustilovsky geodesics, using calculus of variations, already worked out in [8] for the Hofer length functional.

In the future work, we will use this coincidence to extend the virtual Morse theory picture of [7] from very special flag manifolds to general monotone symplectic manifolds.

\section{Statement and proof}

2.1. The group of Hamiltonian symplectomorphisms and Hofer metric. Given a smooth function $H: M^{2 n} \times[0,1] \rightarrow \mathbb{R}$, there is an associated time-dependent Hamiltonian vector field $X_{t}, 0 \leq t \leq 1$, defined by

$$
\omega\left(X_{t}, \cdot\right)=-d H_{t}(\cdot) \text {. }
$$

Received by the editors May 3, 2012. 
The vector field $X_{t}$ generates a path $\gamma:[0,1] \rightarrow \operatorname{Diff}(M)$, starting at $i d$. Given such a path $\gamma$, its end point $\gamma(1)$ is called a Hamiltonian symplectomorphism. The space of Hamiltonian symplectomorphisms forms a group, denoted by $\operatorname{Ham}(M, \omega)$.

In particular, the path $\gamma$ above lies in $\operatorname{Ham}(M, \omega)$. It is well-known that any smooth path $\gamma$ in $\operatorname{Ham}(M, \omega)$ with $\gamma(0)=i d$ arises in this way (is generated by $H: M \times$ $[0,1] \rightarrow \mathbb{R}$ as above). Given a general smooth path $\gamma$, the Hofer length, $L(\gamma)$ is defined by

$$
L(\gamma):=\int_{0}^{1} \max _{M} H_{t}^{\gamma}-\min _{M} H_{t}^{\gamma} d t,
$$

where $H^{\gamma}$ is a generating function for the path $t \mapsto \gamma(0)^{-1} \gamma(t), 0 \leq t \leq 1$. The Hofer distance $\rho(\phi, \psi)$ is defined by taking the infinum of the Hofer length of paths from $\phi$ to $\psi$. We only mention it, to emphasize that it is a deep and interesting theorem that the resulting metric is non-degenerate $(\mathrm{cf} .[1,3])$. This gives $\operatorname{Ham}(M, \omega)$ the structure of a Finsler manifold.

We now consider $L$ as a functional on the space of paths in $\operatorname{Ham}(M, \omega)$ starting at $i d$ and ending at some fixed end points, denote this by $\Omega \operatorname{Ham}(M, \omega)$. It is shown by Ustilovsky that $\gamma$ is a smooth critical point of

$$
L: \Omega \operatorname{Ham}(M, \omega) \rightarrow \mathbb{R},
$$

if there is a unique pair of points $x_{\max }, x_{\min } \in M$ maximizing, respectively minimizing the generating function $H_{t}^{\gamma}$ at each moment $t$, and such that $H_{t}^{\gamma}$ is Morse at $x_{\max }$, $x_{\text {min }}$. We shall call such a $\gamma$ Ustilovsky geodesic.

Consequently, it makes sense to ask for the Morse index of Ustilovsky geodesics, (which might a priori be infinite.) Moreover, it is easy to see that $\operatorname{index}_{L}(\gamma)=$ $\operatorname{index}_{L_{+}}(\gamma)+\operatorname{index}_{L_{-}}(\gamma)$, where:

$$
L_{+}(\gamma):=\int_{0}^{1} \max \left(H_{t}^{\gamma}\right) d t,
$$

for $H_{t}^{\gamma}$ in addition normalized by the condition:

$$
\int_{M} H_{t}^{\gamma} \cdot \omega^{n}=0
$$

The functional $L_{-}$is defined similarly as above. It will be the Morse index of $\gamma$ with respect to $L_{+}$that we compute.

Fix a small $\epsilon, 0<\epsilon<1$, s.t. the linearized flow (isotopy) at $x_{\max }$ of $\left.\gamma\right|_{[0, \epsilon]}$ has no non-trivial periodic orbits with positive period. Let us denote the periodic orbit of the isotopy $\left.\gamma\right|_{[0, \epsilon]}$ associated to $x_{\max }$ by $x_{\max , 0}$, and likewise the periodic orbit of the isotopy $\left.\gamma\right|_{[0,1]}$ associated to $x_{\max }$ by $x_{\max , 1}$. We will say that $\gamma$ is non-degenerate if $x_{\max , 1}$ is non-degenerate in the sense of Floer theory, in other words the time 1 linearized flow at $x_{\max }$ has no non-trivial time 1 periodic orbits.

Theorem 2.1. For $\gamma$ a non-degenerate Ustilovsky geodesics as above, the Morse index of $\gamma$ with respect to $L_{+}$is

$$
\left|C Z\left(x_{\max , 1}\right)-C Z\left(x_{\max , 0}\right)\right| .
$$

Remark 2.2. The above expression is independent of any choices of normalization of $C Z$ index appearing in literature. Moreover, it is precisely the index of the real linear 
Cauchy Riemann operator on which the conjecture is based in [7]. A better way to understand this coincidence is outlined in Section 1.3 of that paper.

Proof. The Morse index theorem [4] cannot be directly applied to

$$
L_{+}=\int_{0}^{1} L(\dot{\gamma}(t), \gamma(t)) d t,
$$

$L(\dot{\gamma}(t), \gamma(t))=\max _{M} H_{t}$, for $H_{t}=\dot{\gamma}(t) \in T_{\gamma(t)} \operatorname{Ham}(M, \omega) \equiv C_{\text {norm }}^{\infty}(M)$, with the latter being smooth functions normalized to have zero mean, (2.3). This is because it clearly does not satisfy the Legendre condition that $\frac{d^{2}}{d \xi^{2}} L(\dot{\gamma}(t), \gamma(t))>0$, for every variation $\xi$ of $\dot{\gamma}$, (for every $t$ ). However, Ustilovsky shows that there is a related functional (actually a quadratic form) $\mathcal{L}_{+}$on the vector space $\Omega_{0} T_{x_{\max }} M$ (based loop space at 0 on the tangent space). With the Hessian of $L_{+}$at $\gamma$ coinciding with the Hessian of $\mathcal{L}_{+}$at 0 , and to which the Morse theorem does apply. This is beautiful, but we refer the reader to [8] and [5, Section 12.4] for further details.

The Morse theorem gives us the following procedure for the calculation of the Morse index of $\gamma$ with respect to $\mathcal{L}_{+}$. Denote by $\gamma_{\tau}$ the restriction of $\gamma$ to $[0, \tau] \subset[0,1]$. Then $\operatorname{index}\left(\gamma_{\tau}\right)$ is a locally constant, lower semi-continuous function in $\tau$, and jumps at a discrete set of $\tau_{i} \in(0,1)$ called conjugate times. The value of the jump mult $\left(\tau_{i}\right)$ is the dimension of the solution space of the associated Jacobi equation. Informally speaking this is dimension of the space of infinitesimal variations of $\gamma_{\tau}$ through extremals with the same endpoints. And a point $\tau \in(0,1]$ is defined to be a conjugate time if this dimension is non-zero.

In the case of the functional $\mathcal{L}_{+}$, it is shown in [8] that $\tau_{0} \in(0,1]$ is a conjugate time if and only if the time $\tau_{0}$ linearized flow of $H$ at the extremizer $x_{\max }$ of $H$ has periodic orbits, and the multiplicity $\operatorname{mult}\left(\tau_{0}\right)$ is the dimension of the space of these periodic orbits.

To keep notation simple, let us denote by $\gamma_{\max }$ the restriction of the linearization of $\gamma$ at $x_{\max }$ to $[\epsilon, 1]$. We will use the construction of Maslov and Conley-Zehnder index given in [6]. For the normalizations used in [6] we show that the absolute value of the Conley-Zehnder index for the path $\gamma_{\max }$ is exactly the Morse index of $\gamma$ for $L_{+}$, from which the statement of the theorem immediately follows by additivity of the Conley-Zehnder/Maslov index with respect to concatenations (and with respect to those normalizations).

Note first that $\gamma_{\max }$ has a crossing at $\tau_{0} \in(0,1)$ with the Maslov cycle if and only if $\gamma_{\max }\left(\tau_{0}\right)$ has 1-eigenvectors, i.e., if and only if $\tau_{0}$ is a conjugate time. Moreover, the dimension of the intersection $I_{\tau_{0}}$ of the diagonal $\Lambda \subset \mathbb{R}^{2 n} \times \mathbb{R}^{2 n}$ with the graph $\operatorname{Gr}\left(\gamma_{\max }\left(\tau_{0}\right)\right)=\left\{\left(z, \gamma_{\max }\left(\tau_{0}\right) z\right) \mid z \in \mathbb{R}^{2 n}\right\}$, is exactly the multiplicity of $\tau_{0}$. The crossing form $Q$ at $\tau_{0}$ can then be identified with the Hessian of $H_{\tau_{0}}^{\gamma}$ at $x_{\max }$, which follows by [6, Remark 5.4]. Since this is non-degenerate by assumption, all the crossings are regular. Our conventions are

$$
\begin{gathered}
\omega\left(X_{H}, \cdot\right)=-d H(\cdot), \\
\omega(\cdot, J \cdot)>0 .
\end{gathered}
$$

Consequently the crossing form is negative definite, and so is negative definite on $I_{\tau_{0}}$. So the signature of $Q$ on $I_{\tau_{0}}$ (number of positive minus number of negative eigenvalues) is just the - mult $\left(\tau_{0}\right)$. The Conley-Zehnder index of $\gamma_{\max }$ is then the sum 
over conjugate times $\tau_{i}$ of $-\operatorname{mult}\left(\tau_{i}\right)$. Consequently Morse index of $\gamma$, is $\left|C Z\left(\gamma_{\max }\right)\right|$.

\section{Acknowledgments}

I would like to thank Leonid Polterovich who gave a crucial initial suggestion, Egor Shelukhin for convincing me to consider the general case, and the anonymous referee for carefully explaining an error regarding normalization in an earlier draft.

\section{References}

[1] H. Hofer, On the topological properties of symplectic maps, Proc. R. Soc. Edinb. Sect. A 115 (1990), 25-38.

[2] Y. Karshon and J. Slimowitz, Shortening the Hofer length of Hamiltonian circle actions (2009), arXiv:0912.0904.

[3] F. Lalonde and D. McDuff, The geometry of symplectic energy, Ann. Math. 141 (2) (1995), 349-371.

[4] M. Morse, The index theorem in the calculus of variations, Duke Math. J. 4 (1938), 231-246.

[5] L. Polterovich, The geometry of the group of symplectic diffeomorphism, Lectures in Mathematics, ETH Zürich, Birkhäuser, Basel, xii, 132 p. sFr. 34.00; DM 44.00; öS 321.00, 2001.

[6] J. Robbin and D. Salamon, The Maslov index for paths, Topology 32 (1993), 827-844.

[7] Y. Savelyev, Virtual Morse theory on $\Omega \operatorname{Ham}(M, \omega)$, J. Differ. Geom. 84 (2010), 409-425.

[8] I. Ustilovsky, Conjugate points on geodesics of Hofer's metric, Differ. Geom. Appl. 6 (1996), $327-342$.

Centre de Recherches Mathématiques, Université de Montréal, C.P. 6128, Succ. Centreville Montréal H3C 3J7, QuéBec, Canada

E-mail address: yasha.savelyev@gmail.com 